\title{
URINARY ALBUMIN TO CREATININE RATIO AS A PREDICTOR OF PRECLAMPSIA AMONG PREGNANT WOMEN AT A TERTIARY CARE HOSPITAL
}

\author{
DR. TEHREEM RAFI, MBBS \\ NISHTAR HOSPITAL, MULTAN, PAKISTAN. \\ DR. ATTIA BATOOL, MBBS \\ NISHTAR HOSPITAL, MULTAN, PAKISTAN. \\ DR. SWAIBA SAMAR, MBBS \\ NISHTAR HOSPITAL, MULTAN, PAKISTAN.
}

\begin{abstract}
:
Objective:_ To determine the diagnostic accuracy of point urinary albumin to creatinine ratio at $20^{+0}$ and $28^{+0}$ weeks of gestation for predicting the possibility of developing pre-eclampsia, late in pregnancy.
\end{abstract}

Study Design: A prospective cross sectional study.

Place and Duration of Study: Gynecology Outpatients Department of Nishtar Hospital, Multan from January 10, 2018 to January 10, 2019.

Methodology: Age, weight, gravidity and Body Mass index was documented for total 250 patients included for
study. Patients were divided on the basis of development of pre-eclampsia and their mean Albumin to creatinine
ratio was compared by applying Student's t-test considering $\mathrm{p} \leq 0.05$ statistically significant. Test positive and
test negative patients were noted. The association of urinary albumin to creatinine ratio with pre-eclampsia was
calculated. SPSS v. 23 software was used to analyze the data.

Results: Mean urinary albumin to creatinine ratio was $22.77 \pm 12.42 \mathrm{mg} / \mathrm{mmol}$ in normotensive women and $46.87 \pm 15.13 \mathrm{mg} / \mathrm{mmol}$ in pre-eclamptic women which was significantly higher in pre-eclamptic patients $(\mathrm{p}<0.001)$. The sensitivity of point urinary albumin to creatinine ratio at a cutoff value of $35.5 \mathrm{mg} / \mathrm{mmol}$ is $73.91 \%$; the specificity is $91.56 \%$; the positive predictive value (PPV) is $72.34 \%$; and negative predictive is $92.16 \%$.

Conclusion: Our study concluded that solitary point urinary albumin creatinine ratio values equal to or higher than $35.5 \mathrm{mg} / \mathrm{mmol}$, when quantified early during the second trimester had a higher specificity than the sensitivity and it can be safely used to rule out the possibility of developing pre-eclampsia, late in pregnancy.

Keywords: Albumin to Creatinine Ratio, Pre-eclampsia, Normotensive, Point Urinary .

DOI: $10.7176 / \mathrm{JMPB} / 62-12$

Publication date: December $31^{\text {st }} 2019$

\section{Introduction:}

In pregnancy, pre-eclampsia is one of the major health issues ${ }^{1}$. According to an estimate, $3-8 \%$ percent of all the pregnancies are complicated and result in a significant rise in perinatal maternal morbidity and mortality ${ }^{2}$. It is the $2^{\text {nd }}$ highest reason of maternal death, blamable for $11.1-17.4 \%$ deaths related to pregnancy ${ }^{3}$. Pre-eclampsia is described as a minimum blood pressure of $140 / 90 \mathrm{mmHg}$ documented on at least two discrete times, minimum of four hours apart, concomitant with at least $300 \mathrm{mg}$ of protein in a twenty four hours urine collection which occurs de novo after twentieth gestational week in a previously normotensive woman and it resolves fully after sixth week in postpartum period ${ }^{4,9}$. Pre-eclampsia can occur in a patient who is suffering from prolonged hypertension before the pregnancy, as well. Abnormal immunological interactions, genetic predilection, endothelial injury and dysfunction are believed to play a noteworthy part in its occurrence but the precise cause is not known, so far ${ }^{5}$. Primary pregnancy, multi-parity, previous history of pre-eclampsia, body mass index 
$\geq 35 \mathrm{~kg} / \mathrm{m}^{2}$, familial history of pre-eclampsia, old age i.e. 40 years or above and multiple pregnancy are some of the danger factors for the development of pre-eclampsia ${ }^{6,10}$. Certain basic medical disorders for example chronic hypertension, chronic kidney disease, diabetes mellitus and anti-phospholipid antibodies syndrome are also thought to intensify the possibility of pre-eclampsia ${ }^{10}$.

Knowledge about the physiology and pathology of pre-eclampsia is defective. Genetic predilection is a key part player as a threefold to fourfold increase in the chances of pre-eclampsia in first degree relative of pre-eclamptic patient. The suggested process of pre-eclampsia begins with inadequate incursion, of maternal vascular bed, by trophoblastic cells due to any anomalous immunological reaction ${ }^{7,8,9}$ which results in hypo-perfusion of placenta and leads to wide-ranging maternal endothelial dysfunction as well as increased vascular permeability. In normal pregnancy, there is peripheral vasodilation but in pre-eclampsia, there is occurrence of vasoconstriction which leads to increase in intra-vascular pressure which together with increased vascular permeability results in generalized body edema. Glomeruloendotheliosis is a condition which arises in kidneys and is rather specific for pre-eclampsia ${ }^{9}$. There is concomitant impairment of glomerular filtration system and intermediate size proteins are lost in urine e.g. albumin and transferrin etc.

A fair number of pregnancies are complicated by pre-eclampsia. There is necessity to define some screening methods to expect the occurrence pre-eclampsia to reduce the risk of perinatal maternal morbidity and mortality along with fetal problems. This can support to monitor and develop the quality of medical services in time afore pre-eclampsia arises and evolves into eclampsia ${ }^{10,11}$. Benefit of the prophylactic administration of aspirin ${ }^{12}$, heparin and calcium in high-risk patients ${ }^{13}$ has been supported by some evidence. A few studies have been performed to define some sort of screening method to predict the risk of pre-eclampsia but outcomes have not been satisfactory, so far. Persistent micro albuminuria is indicative of huge possibility of impairment of the filtration capability of the glomeruli of the kidney, and it is of great importance as a likely forecaster of preeclampsia in pregnancy. Current study was done to analyze the usefulness of spot urinary albumin to creatinine ratio during early pregnancy, in asymptomatic pregnant women, to determine the likelihood of threat of preeclampsia.

\section{Material and methodology:}

This is a prospective cross sectional study conducted from January 10, 2018 to January 10, 2019. The data was collected in Gynecology Outpatients Department of Nishtar Hospital, Multan, after obtaining ethical approval from the hospital ethical committee. The pregnant women patients who presented to the gynecology outdoor with singleton pregnancies at gestational age of 20 to 28 weeks were chosen using non probability consecutive sampling technique. Only those patients who were above eighteen years of age and had no proteinuria on dipstick test at the time of presentation were included in the study. A total of 250 patients were selected and informed consent was obtained from all of them, on written forms. Fifty patients could not follow up, mostly because of being from distant residential areas. All the patients were properly examined after history taking. Age, weight, gravidity, Body Mass index was documented. Risk factors of pre-eclampsia were ruled out. A point midstream urinary sample was gathered for the analysis. Albumin level was estimated using immuneturbidimetric microalbumin method. Modified Jaffe's technique in ERBA XL640 was used to estimate creatinine. Commercially available reagents were used. All the patients were followed up till the time of delivery. Blood pressure was measured and the patients were assessed for the occurrence of signs and symptoms of pre-eclampsia for example edema, epigastric pain, nausea, vomiting, visual disturbances and altered urine output, at the time of each visit. A minimum blood pressure of $140 / 90 \mathrm{mmHg}$ along with proteinuria is the definition of pre-eclampsia. As in our reference study conducted by Mishra VV et al. ${ }^{14}, 35.5 \mathrm{mg} / \mathrm{mmol}$ was taken as cutoff value for Albumin to creatinine ratio. The values for Albumin to creatinine ratio was considered testpositive if they were equal to or more than $35.5 \mathrm{mg} / \mathrm{mmol}$. The values below this level were considered testnegative. Patients who did not consent or had multiple pregnancy, proteinuria at dip-stick, hematuria, chronic hypertension, acute renal failure, chronic kidney disease, current urinary tract infection, and/or diabetes mellitus were excluded from our study.

All the data was collected by the researcher himself on a preformed Performa. Patients were divided on the basis of development of pre-eclampsia and their mean Albumin to creatinine ratio was compared by applying Student's t-test, considering $\mathrm{p} \leq 0.05$ statistically significant. Test positive and test negative patients were noted. The association of Albumin to creatinine ratio with pre-eclampsia was calculated. SPSS v.23 software was used to analyze the data. 


\section{Results:}

We enrolled 200 patients in our study. The mean age of all the patients was $27.50 \pm 5.78$ years and mean body mass index was $28.63 \pm 5.03 \mathrm{~kg} / \mathrm{m}^{2}$. Ninety three $(46.5 \%)$ patients were primigravida and one hundred and seven $(53.5 \%)$ patients were multigravida. Mean systolic blood pressure in the normotensive patients and pre-eclamptic patients was $115.35 \pm 4.78 \mathrm{mmHg}$ and $143.27 \pm 3.38 \mathrm{mmHg}$, respectively. Mean diastolic blood pressure in the normotensive patients and pre-eclamptic patients was $81.78 \pm 3.37 \mathrm{mmHg}$ and $95.48 \pm 2.65 \mathrm{mmHg}$, respectively.

Mean urinary albumin to creatinine ratio was $22.77 \pm 12.42 \mathrm{mg} / \mathrm{mmol}$ in normotensive women and $46.87 \pm 15.13$ $\mathrm{mg} / \mathrm{mmol}$ in pre-eclamptic women. This value was significantly higher in pre-eclamptic patients $(\mathrm{p}<0.001)$. According to our reference study ${ }^{14}$, the cutoff value for urinary albumin to creatinine ratio was taken as 35.5 $\mathrm{mg} / \mathrm{mmol}$. (Table-I)

Over all, the test was positive in forty seven (23.5\%) patients and negative in the remaining one hundred and fifty three $(76.5 \%)$ patients (Table-II). Table-III represents the association of albumin to creatinine ratio and preeclampsia. This shows that out of total forty seven $(23.5 \%)$ test-positive patients, thirty four $(17 \%)$ developed pre-eclampsia in later stages of pregnancy and thirteen $(6.5 \%)$ stayed normotensive. In test-negative patients, 12 $(6 \%)$ developed pre-eclampsia in later stages of pregnancy, while $141(70.5 \%)$ stayed normotensive. The sensitivity of point urinary albumin to creatinine ratio at a cutoff value of $35.5 \mathrm{mg} / \mathrm{mmol}$ is $73.91 \%$; the specificity is $91.56 \%$; the positive predictive value (PPV) is $72.34 \%$; and negative predictive is $92.16 \%$.

\section{Table-I}

\section{Comparison of urinary Albumin Creatinine Ratio (ACR) between normotensive and preeclamptic patients}

\begin{tabular}{|l|c|c|}
\hline \multicolumn{1}{|c|}{ ACR (mg/mmol) } & Normotensive (n=154) & Pre-eclamptic (n=46) \\
\hline Mean \pm S.D & $22.77 \pm 12.42$ & $46.87 \pm 15.13$ \\
\hline Range & $3.90-47.88$ & $19.80-88.78$ \\
\hline Median & 24 & 47 \\
\hline p-value & \multicolumn{2}{|c|}{$<0.001$} \\
\hline \multicolumn{2}{|c|}{$\mathrm{P}<0.001=$ statistically significant. }
\end{tabular}

Table-II

Distribution of women according to the positivity of the test

\begin{tabular}{|c|c|c|c|}
\hline Test Used & Test Positive & Test Negative & Total \\
\hline ACR $(\mathrm{mg} / \mathrm{mmol})$ & $47(23.5)$ & $153(76.5)$ & $200(100)$ \\
\hline
\end{tabular}

Data are presented as Number (percentage); $\mathrm{ACR}=$ Albumin Creatinine Ratio.

Table-III

Association of Albumin Creatinine Ratio with pre-eclampsia

\begin{tabular}{|c|c|c|c|}
\hline Urinary ACR & Normotensive & Pre-eclamptic & Total \\
\hline Test Positive & $13(6.5)$ & $34(17)$ & $47(23.5)$ \\
\hline Test negative & $141(70.5)$ & $12(6)$ & $153(76.5)$ \\
\hline Total & $154(77)$ & $46(23)$ & $200(100)$ \\
\hline
\end{tabular}

Data are presented as Number (percentage); $\mathrm{ACR}=$ Albumin Creatinine Ratio.

\section{Discussion:}

We witnessed in our study that the many of the patients who had high albumin to creatinine ratio, observed while they were asymptomatic, developed pre-eclampsia; whereas most of those who were test-negative, did not. This test had higher specificity and negative predictive values as compared with the sensitivity and positive predictive 
values. Spot urinary albumin creatinine during $2^{\text {nd }}$ trimester of pregnancy is a better tool to rule out the risk of pre-eclampsia. Having a small dissimilarity with our results, according to Mishra VV ${ }^{14}$ et al, this test had a higher specificity as well as sensitivity and predicted pre-eclampsia with sensitivity of $87.5 \%$ and specificity of $96.30 \%$, long before the development of clinical signs and symptoms. They concluded that the test was sufficient enough to predict the possibility of pre-eclampsia. Although their inference was comparable with the ones presented by Baweja S. et al. ${ }^{15}$ (83.3\% sensitivity) and Fatema K et al. ${ }^{16}$ (80\% sensitivity), but the study consisted of a very small sample size.

A arbitrary urinary protein to creatinine ratio is equally good as the protein content in twenty four hour collection of urine; and it is good enough to screen the patients at risk of pre-eclampsia for significant proteinuria, prior to development of clinical manifestation ${ }^{17}$. Nisell $\mathrm{H}$ et al. ${ }^{18}$ stated that a single sample albumin to creatinine ratio was no different from 24 hours urine collection, rather 24 hour collection of the urine is tiring for both the patient as well as the handling staff. Similar study was conducted by Cade TJ et al. ${ }^{19}$ and they came to the conclusion that the protein creatinine ratio, at cutoff point $30 \mathrm{mg} / \mathrm{mmol}$, was considerably effective to clear the at-riskpatients of significant proteinuria. Owing to the effectiveness of random urinary protein creatinine ratio, 24-hour urinary collection is seldom recommended. Singh R et al. ${ }^{20}$ studied one hundred and forty four normotensive pregnant women in their twenty fourth to twenty eighth week of gestation. They found out that the microalbuminuria in second trimester was highly linked with development of pre-eclampsia, late in pregnancy, apart from its association with low birth weight babies and preterm delivery.

Poon LC et al. ${ }^{21}$ conducted a study on a large group of pregnant women between their $11^{\text {th }}$ and $13^{+6}$ weeks of gestation. Their results were in contradiction with ours. They found positive protein urea in $55 \%$ of the normotensive patients and $75 \%$ of the pre-eclamptic patients. The protein to creatinine ratio did not give any fruitful results in the estimation of the probability of pre-eclampsia. The reason behind these results might be that the patients were observed too early, much before the age of viability of the fetus.

\section{Conclusion:}

Our study concluded that single point urinary albumin creatinine ratio values of equal to or higher than $35.5 \mathrm{mg} / \mathrm{mmol}$, when quantified early during the second trimester, were good enough to expect pre-eclampsia before the onset of clinical signs, having $73.91 \%$ sensitivity and $91.56 \%$ specificity. Therefore it can be safely said that a positive test can expect the incidence of pre-eclampsia to a great extent, but a negative test rules out this possibility to a much greater extent.

\section{Conflict of interest: NIL}

Funding Source: NIL

\section{References:}

1. Tranquilli AL, Brown MA, Zeeman GG, Dekker G, Sibai BM. The definition of severe and early-onset preeclampsia. Statements from the International Society for the Study of Hypertension in Pregnancy (ISSHP). Pregnancy Hypertens. 2013;3(1):44-7.

2. Anderson UD, Olsson MG, Kristensen KH, Åkerström B, Hansson SR. Review: biochemical markers to predict preeclampsia. Placenta. 2012;33(Suppl):S42-S47.

3. Say L, Chou D, Gemmill A, Tunçalp Ö, et al. Global causes of maternal death: a WHO systematic analysis. The Lancet. 2014;2(6):e323-33.

4. Mol BW, Roberts CT, Thangaratinam S, Magee LA, De Groot CJ, Hofmeyr GJ. Pre-eclampsia. The Lancet. 2016 11;387(10022):999-1011.

5. Lin S, Leonard D, Co MA, et al. Pre-eclampsia has an adverse impact on maternal and fetal health. Translational Research. 2015;165(4):449-63.

6. Myers JE, Kenny LC, et al. Angiogenic factors combined with clinical risk factors to predict preterm pre-eclampsia in nulliparous women: a predictive test accuracy study. BJOG-Int J Obstet Gynecol. 2013;120(10):1215-23.

7. Saito S, Nakashima A. A review of the mechanism for poor placentation in early-onset preeclampsia: the role of autophagy in trophoblast invasion and vascular remodeling. J. Reprod. Immunol. 2014;101:80-8. 
8. Perez-Sepulveda A, Torres MJ, Khoury M, Illanes SE. Innate immune system and preeclampsia. Front Immunol. 2014;5:244.

9. Chaiworapongsa T, Chaemsaithong P, Yeo L, Romero R. Pre-eclampsia part 1: current understanding of its pathophysiology. Nat Rev Nephrol. 2014;10(8):466-80.

10. Ukah UV, Payne B, Côté AM, Hoodbhoy Z, von Dadelszen P. Risk factors and predictors of preeclampsia. The FIGO Textbook of Pregnancy Hypertension. GLOWM. 2016:75-100.

11. Kane SC, Da Silva Costa F, Brennecke SP. New directions in the prediction of pre-eclampsia. Aust Nz J Obstet Gyn. 2014;54(2):101-7.

12. Bujold E, Roberge S, Lacasse $Y$, et al. Prevention of preeclampsia and intrauterine growth restriction with aspirin started in early pregnancy: a meta-analysis. Obstet Gynecol. 2010;116(2, Part 1):402-14.

13. de Vries JI, van Pampus MG, Hague WM, Bezemer PD, Joosten JH. Low-molecular-weight heparin added to aspirin in the prevention of recurrent early-onset pre-eclampsia in women with inheritable thrombophilia: the FRUIT-RCT. J Thrombos Haemost. 2012;10(1):64-72.

14. Mishra VV, Goyal PA, et al. Evaluation of Spot Urinary Albumin-Creatinine Ratio as Screening Tool in Prediction of Pre-eclampsia in Early Pregnancy. J Obstet Gynecol India. 2017;67(6):405-8.

15. Baweja S, Kent A, Masterson R, Roberts S, McMahon LP. Prediction of pre-eclampsia in early pregnancy by estimating the spot urinary albumin: creatinine ratio using high-performance liquid chromatography. BJOG-Int J Obstet Gynecol. 2011;118(9):1126-32.

16. Fatema K, Khatun M, Akter S, Ali L. Role of urinary albumin in the prediction of preeclampsia. Faridpur Med Col J. 2011;6(1):14-8.

17. Sanchez-Ramos L, Gillen G, Zamora J, Stenyakina A, Kaunitz AM. The protein-to-creatinine ratio for the prediction of significant proteinuria in patients at risk for preeclampsia: a meta-analysis. Ann Clin Lab Sci. 2013;43(2):211-20.

18. Nisell H, Trygg M, Back R. Urine albumin/creatinine ratio for the assessment of albuminuria in pregnancy hypertension. Acta Obstet Gynecol. 2006;85:1327-30.

19. Cade TJ, Gilbert SA, Polyakov A, Hotchin A. The accuracy of spot urinary protein-to-creatinine ratio in confirming proteinuria in pre-eclampsia. Aust Nz J Obs Gynaecol. 2012;52(2):179-82.

20. Singh R, Tandon I, Deo S, Natu SM. Does microalbuminuria at mid-pregnancy predict development of subsequent pre-eclampsia?. J Obstet Gynaecol. 2013;39(2):478-83.

21. Poon LC, Kametas N, Bonino S, Vercellotti E, Nicolaides KH. Urine albumin concentration and albumin-to-creatinine ratio at $11+0$ to $13+6$ weeks in the prediction of pre-eclampsia. BJOG-Int $\mathrm{J}$ Obstet Gynecol. 2008;115(7):866-73. 